\title{
Non-Antibody Mediated Roles of B Cells in Allograft Survival
}

\author{
Geetha Chalasani • David Rothstein
}

Published online: 29 June 2014

(C) Springer International Publishing AG 2014

\begin{abstract}
Antibody production is unquestionably a key effector function of $\mathrm{B}$ cells that remains a formidable barrier against long-term graft survival. However, emerging evidence indicates that B cells play a key role in shaping the effector responses by mechanisms that extend beyond their function as antibody producing cells. B cell depletion in transplant recipients has resulted in paradoxical outcomes of increased graft rejection versus improved graft function, implying that $\mathrm{B}$ cells function as both enhancers and regulators of the alloimmune response. Based on findings from animal and human studies, we address mechanisms by which B cells modulate the immune response and highlight their role in promoting allograft rejection or tolerance.
\end{abstract}

Keywords Effector B cells $\cdot$ Antibody-independent $\cdot$ Antigen presentation · Transplant rejection · Regulatory B cells . TNF $\alpha \cdot$ IL-10 · TIM-1 · Transplant tolerance $\cdot$ Allograft survival

G. Chalasani $\cdot$ D. Rothstein

Departments of Medicine (Renal-Electrolyte), University of Pittsburgh School of Medicine, Pittsburgh, PA, USA

D. Rothstein

Surgery, University of Pittsburgh School of Medicine, Pittsburgh, PA, USA

G. Chalasani $\cdot$ D. Rothstein

Immunology, University of Pittsburgh School of Medicine, Pittsburgh, PA, USA

G. Chalasani $\cdot$ D. Rothstein $(\bowtie)$

Thomas E. Starzl Transplantation Institute, University of Pittsburgh School of Medicine, 200 Lothrop Street, W1545 Biomedical Science Tower, Pittsburgh, PA 15261, USA

e-mail: rothsteindm@upmc.edu

\section{Introduction}

Classically, $\mathrm{T}$ cells mediate the adaptive cellular immune response and provide helper function to B cells, allowing them to mount a humoral response. In addition to their critical function in host defense, cytotoxic antibodies can play a pathological role targeting parenchymal tissues in autoimmune disease and organ transplantation [1-4]. Based on this paradigm, T cell-directed therapies have been the mainstay of immunosuppressive therapy in transplantation, aiming to prevent both cellular and antibody-mediated rejection. The role of B cells and antibodies has received renewed interest as improved detection and diagnosis reveal that donor-specific antibodies significantly contribute to both acute and chronic allograft loss despite potent immunosuppression $[5,6]$.

Over two decades ago, B cells were shown to also be able to directly influence $\mathrm{T}$ cell responses through their ability to present antigen, provide co-stimulation and produce cytokines [7-11]. However, a major role for $B$ cells in the $T$ cell response was not widely accepted in the field, because such a small fraction of B cells express cytokines and initial studies relied heavily on congenitally B-deficient mice whose defects in lymphoid architecture could directly affect $\mathrm{T}$ cell responses. Nonetheless, recent findings in both humans and rodent models have re-energized the field and now provide strong evidence that $\mathrm{B}$ cells and their cytokines play a significant role in modulating cellular immune responses. First, the use of bone marrow chimeras and mice carrying specific gene knockouts in B cells definitively demonstrates that B cells can either augment or inhibit $\mathrm{T}$ cell-mediated immune responses $[11-13,14 \cdot \bullet]$. Second, with the advent of Rituximab (anti-CD20), efficient and specific B cell depletion in humans has become a reality. Studies using Rituximab show that B cell depletion is effective in treating autoimmune diseases, including multiple sclerosis (MS,) rheumatoid arthritis (RA), and type I diabetes (T1D), that are either T cell-mediated or 
demonstrate that treatment is effective without any measurable decrease in antibody levels [11, 14••, 15, 16]. Acute antibody-mediated depletion of B cells in wild-type mice corroborates the ability of B cells to either augment or inhibit $\mathrm{T}$ cell responses in various inflammatory settings $[1,12,17$, 18・•, 19-21]. While progress has been made identifying their role, our inability to specifically identify rare proinflammatory "Beff" cells exhibiting effector function, or equally rare regulatory B cells (Bregs), remains a major barrier (Fig 1). In the face of therapeutic agents that target $B$ cells, identification of pro- versus anti-inflammatory subsets of B cells and their exact roles, gains even more importance. Here we will review the evidence in mice and humans, for Beff and Breg cells, and their role in modulating the immune response, with a focus on recent findings pertaining to transplantation.

\section{Antibody-Independent Functions of B Cells in Driving T Cell Responses and Graft Rejection}

The mature B cell population consists of innate-like B cells (B1 and Marginal Zone, MZ) and follicular (FO) B cells that have diverse functions in immune responses [22]. Innate-like $\mathrm{B}$ cells produce natural antibodies to carbohydrate and phospholipid antigens independent of T-cell help (Thymus-independent antigens, e.g., pneumococcal capsular polysaccharides, blood group antigens, phosphatidylcholine, etc.) [22]. These IgM antibodies form immune complexes that modulate DC maturation and contribute to self-tolerance, anti-microbial immunity, and autoimmunity [23, 24]. Follicular B cells respond to protein antigens and depend on T-cell help to mediate
IgG responses (Thymus-dependent antigens, e.g., HLA, ovalbumin, etc) [25]. In addition to BCRs, B cells express multiple TLRs and integrate signals derived from PAMPs/endogenous TLR ligands and antigen recognition [26-29]. TLR ligands (e.g., LPS, CpG DNA) either alone (innate-like B cells) or in concert with antigen (FO B cells) can activate B cells leading to rapid antibody responses and upregulated expression of molecules such as MHC II, CD40, CD80, CD86, CD70, OX40L, and ICOSL that facilitate interactions with $\mathrm{T}$ cells [22, 28, 30, 31]. Also, TLR- and/or BCR-derived signals induce B cell expression of cytokines such as IL-10, IL-6 and IFN $\gamma$ that can influence differentiation of T cells [31].

Alloantibodies produced by B cells function as mediators of graft injury via ADCC, endothelial injury, and activating NK cells [32-34]. In addition, alloantibodies contribute to effective T cell priming by APCs, presumably through Fc receptor-mediated pathways, against alloantigens and other linked antigens expressed on the graft [35]. As detailed below, recent studies have shown that $\mathrm{B}$ cells also enhance $\mathrm{T}$ cell responses in the context of transplantation via mechanisms other than antibody production.

\section{Antigen Presentation by B Cells in Transplant Rejection}

$B$ cells are most efficient at presenting antigen taken up via the BCR, while internalized non-specific antigen bound to complement and $\mathrm{Fc}$ receptors can also be presented, albeit less efficiently [36-39]. The role of B cells in T cell priming has been controversial and was thought to be limited to reactivation of memory $\mathrm{T}$ cells $[40,41]$. Initial reports of impaired CD4 $\mathrm{T}$ cell responses in $\mathrm{B}$ cell-deficient mice were
Fig. 1 Properties of Effector (Beff) and Regulatory (Breg) B cells. This representation lists key cytokines, costimulatory molecules, and antigenpresenting functions shown to play an important role in the function of Beff and Bregs. While identification of phenotype of Beff cells remains unclear, B cell subsets contributing to Breg function are listed. $M Z$ marginal zone, TIM-1 (T cell Ig and Mucin domain-1 molecule)

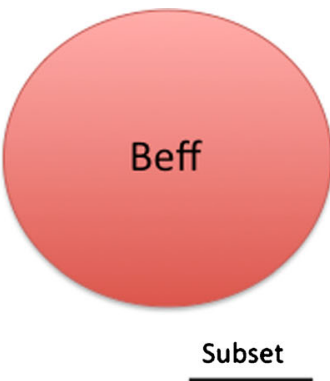

Cytokines

$$
\begin{aligned}
& \text { IFNy } \\
& \text { TNF } \alpha \\
& \text { IL-6 } \\
& \text { IL-17 }
\end{aligned}
$$

?

IL-10

IL-35
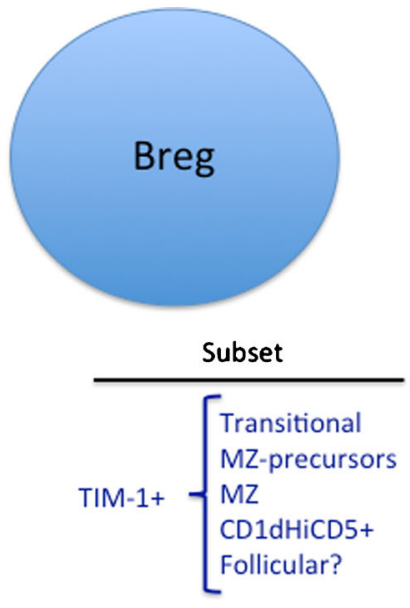

Plasmablasts

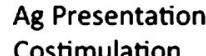

Costimulation
MHC II, MHC

CD80/CD86

$\mathrm{CD} 40$

OX40L

ICOSL 
confounded by inherent disruption of lymphoid architecture, loss of follicular DCs, and decreased T cell numbers [42-45]. However, recent studies in multiple-model antigen systems with intact B cell compartment and/or preserved lymphoid architecture, ranging from infections (bacterial, fungal, parasitic, and viral) to autoimmunity, have now established B cells as critical APCs for CD4 and CD8 T cell responses [46-51] (Fig. 1).

It was recognized from earlier studies that heart allograft rejection was delayed in B cell-deficient mice, although this was largely attributed to the lack of antibodies since antibody transfer experiments restored rejection [45,52]. However, subsequent studies from our and other groups have shown that alloreactive $\mathrm{T}$ cell memory is impaired in B cell-deficient mice, suggesting a role for B cells beyond antibody production $[35,53,54]$. Alloreactive effector $\mathrm{T}$ cells developing in the absence of B cells contained fewer IL7R $\alpha$ - expressing memory precursors, resulting in decreased memory $\mathrm{T}$ cell numbers and impaired memory recall [54]. In B cell-T cell cotransfer experiments, adoptive transfer of primed B cells enhanced proliferation of activated CD4 $\mathrm{T}$ cells and upregulated expression of anti-apoptotic Bcl2 in CD8 T cells, resulting in increased memory $\mathrm{T}$ cell generation [54]. These findings are consistent with observed results in LCMV and L. monocytogenes infections, which showed exaggerated contraction of effector T cells and attenuated T cell memory in the absence of B cells [46, 51].

Noorchashm et al. were the first to demonstrate the role of $\mathrm{B}$ cells as APCs in allograft rejection [55]. Mice lacking the ability of B cells to present antigen to CD4 T cells (B cellspecific impairment in either MHC II expression or MHC II peptide loading) showed attenuated donor-reactive CD4 T cell proliferation and IgG antibodies, significantly delaying acute rejection of heart allografts [55]. Despite these compelling results, antibody responses were also attenuated in the absence of B-APC function. Thus, it remained unresolved whether B cells provided requisite help for $\mathrm{T}$ cell responses in transplantation independent of antibody production. We addressed this question in a recent study using murine models of chronic allograft vasculopathy since antibodies are important for pathogenesis of chronic rejection [56•]. Chronic rejection was attenuated in mice that lacked both $\mathrm{B}$ cells and antibodies ( $\mu \mathrm{MT}$ [57]). However, mice containing B cells that expressed surface BCR but could not secrete antibodies (AID/ $\mu$ sKO [58]) developed chronic rejection comparable to wild-type controls. Adoptive transfer of B cells from AID/ $\mu$ sKO into $\mu \mathrm{MT}$ mice recapitulated chronic rejection in the complete absence of circulating antibodies. This conclusively demonstrated that B cells were sufficient and secreted antibodies were not necessary for pathogenesis of chronic rejection. Moreover, cytokine-producing alloreactive $\mathrm{T}$ cells and $\mathrm{T}$ cell infiltration of allograft vessels were also diminished in the absence of B cells, but were restored by antibody-deficient B cells [56•]. Thus, B cells influence $\mathrm{T}$ cell responses via antibody-independent mechanisms promoting chronic rejection.

We next examined whether antigen recognition by B cells (cognate) vs. antigen-independent (non-cognate) mechanisms were providing help for T cells. Using BCR-tg mice, we found that non-cognate $\mathrm{B}$ cells could only partially restore chronic rejection and alloreactive $\mathrm{T}$ cell responses despite normal lymphoid architecture, suggesting that cognate functions of $\mathrm{B}$ cells were also required [56 ${ }^{\bullet}$. The cognate role of B cells as APCs was further tested in mice containing B cells that lacked MHC I and II expression, and could not present antigen to CD4 and CD8 T cells, respectively. Despite preserved lymphoid architecture, alloreactive CD4 and CD8 T cell cytokine production and chronic rejection were attenuated in the absence of antigen presentation by B cells. Thus, B cells function as key APCs to present cognate antigen, and this is required to drive $\mathrm{T}$ cell responses causing chronic rejection.

In conjunction with B - APC function, CD40 and CD80/86 expression on $\mathrm{B}$ cells contributes to anti-pneumocystis and arthritogenic $\mathrm{CD} 4 \mathrm{~T}$ cell responses $[47,59]$. In addition to cognate antigen presentation, the ability of $\mathrm{B}$ cells to present non-cognate antigen internalized via $\mathrm{Fc}$ or complement receptor-mediated uptake, overcomes the limitation of low frequencies of antigen-specific B cells [38, 39]. B1 and MZ $\mathrm{B}$ cells constitutively express CD80 and CD86, and present antigen efficiently to $\mathrm{T}$ cells [60-62] and it remains to be tested if these B cells exhibit superior APC function than FO $\mathrm{B}$ cells in alloimmune responses. Also, determining whether $\mathrm{B}$ cells are influencing CD8 T cell responses in transplantation by enabling CD4 $\mathrm{T}$ cell help or by cross-presenting antigen to CD8 T cells needs to be explored in future studies.

\section{Tertiary Lymphoid Organs in Transplant Rejection}

B cells influence the formation of tertiary lymphoid structures found at sites of inflammation that facilitate in-situ antibody and T cell responses [63]. Tertiary lymphoid organs are found in grafts undergoing chronic rejection and support the development of alloantibodies, and of alloreactive effector and memory $T$ cells [64-66]. Indeed, abrogation of tertiary lymphoid organ formation by LT $\beta$ R-Ig treatment after transplantation inhibits $\mathrm{B}$ cell responses and prevents chronic rejection [67].

Additional mechanisms by which B cells contribute to immune responses that are yet to be explored in transplantation include costimulation, effector cytokine production, and modulation of innate responses. B cells express several costimulatory ligands such as ICOSL, OX40L, 41BBL, and CD70 that provide late costimulatory signals important for $T$ cell differentiation to memory cells, and can function as potent costimulators of $\mathrm{T}$ cells $[30,68,69]$. Indeed, B cell-specific expression of OX40L was shown to be important for effector 
differentiation and memory development of OT-II TCR-tg T cells [68]. Cognate and non-cognate B cells also influence the generation and expansion of $\mathrm{T}_{\mathrm{FH}}$ cells by providing costimulation via ICOSL $[70 \bullet \bullet, 71 \bullet, 72 \bullet]$. In addition to antigen presentation and costimulation, effector cytokines secreted by $\mathrm{B}$ cells play an important role in shaping $\mathrm{T}$ cell responses in infections and autoimmune diseases [11]. IL-2 from B cells was essential for protective Th2 memory T cells in H. polygyrus infection [13]. B cell TNF $\alpha$ was important for protective antibodies in $H$. polygyrus and the expansion of Th1 cells in $T$. gondii infections $[13,73]$. IL-6 from B cells following Salmonella infection and EAE induction stimulated CD4 T cell IL-17 production [14••, 50]. Beyond shaping adaptive $T$ cell responses, $B$ cells also play an important role in modulating innate immune responses by mechanisms other than antibody production. GM-CSF secreted by B cells was shown to be essential for neutrophil phagocytosis, bacterial clearance, and survival in sepsis [74••]. IL-12 production by DCs is enhanced in the absence of B cells shifting the balance toward Th1 responses due to lack of B cell IL-10 [75]. CCL7 from B cells guides monocyte infiltration into myocardium after myocardial infarction, exacerbating injury that is attenuated with $\mathrm{B}$ cell depletion [76••]. How these costimulatory molecules and cytokines from B cells shape the alloimmune response and influence graft survival remains to be explored. Future studies need to understand how and when to intervene to selectively target effector B cells and alter the course of alloimmune response to prevent rejection.

\section{Regulatory B Cells: Lessons from Murine Models}

Reports that B cells can inhibit T cell responses date back four decades [77]. However, a regulatory role for B cells did not begin gaining traction until the early 2000s when more definitive studies were performed. Mizoguchi and Bhan first coined the term "regulatory B cells" when they noted that spontaneous colitis developing in dysregulated TCR $\alpha-/-$ mice was significantly worse when crossed onto a B-deficient ( $\mu \mathrm{MT}$ ) background [78]. They subsequently demonstrated that colitis in TCR $\alpha-/-$ mice led to an increase in CD1d + (marginal zone) $B$ cells expressing IL-10, which could inhibit colitis when transferred into B-deficient $\mu \mathrm{MT}$.TCR $\alpha-/-$ mice, and this inhibition was IL-10-dependent [79] (Fig 1). Around the same time, Fillatreau explained earlier observations that B-deficient $(\mu \mathrm{MT})$ mice developed aggressive EAE (a murine model of MS) by showing that B cells ameliorated the disease by producing IL-10 [12, 80]. This was done by generating mixed bone marrow chimeras in $\mu \mathrm{MT}$ mice so that only the $B$ cells lacked IL-10. In contrast to control chimeras with wildtype B cells, mice whose B cells lacked IL-10 exhibited aggressive, non-remitting EAE, as seen in B-deficient mice.
These experiments clearly attributed Breg activity to IL-10 expression, and moreover, the latter study addressed the role of Bregs in intact mice whose splenic architecture and B cell compartment were restored by bone marrow transplantation.

An immunoregulatory role for B cells has since been supported by the demonstration that acute depletion or deficiency of B cells can worsen disease, while transfer of B cells (or subpopulations) from diseased or immunized mice can suppress inflammatory responses in a variety of experimental models including rheumatoid arthritis (collagen-induced arthritis), contact hypersensitivity, type-1 diabetes, SLE, allergic airway disease, and allogeneic transplantation [18••, 21, 81-85]. In almost every case, Breg activity is IL-10 dependent, although this does not preclude a role for other mechanisms [10, 18••, 83, 86]. In EAE and SLE, B cell depletion before disease onset worsens disease, whereas depletion once disease has been induced ameliorates disease [1, 87]. These studies suggest that Bregs may play a dominant role during disease initiation, while B cells have a predominant effector/ APC function during disease progression.

Phenotypic Identification of Bregs Remains a Major Problem in the Field:

The studies above demonstrate that Bregs inhibit disease in an IL-10-dependent manner in multiple disease models. Thus, expression of IL-10 has become the sine qua non for identification of Bregs. Despite this, for reasons detailed below, there remains no way to specifically identify Bregs based on phenotype, and this may be the single largest barrier to further understanding Breg immunobiology. Unfortunately, IL-10 is not observed in situ, or in freshly isolated B cells. Rather, IL10 expression is induced after activation of $\mathrm{B}$ cells in vitro with potent mitogenic stimuli. Thus, identification of other markers is essential, but thus far, none are specific. In earlier studies, B cells were stimulated in culture for up to $72 \mathrm{~h}$ with CD40-ligation and/or TLR-ligands, and such conditions alter phenotype. Refinements in cytokine detection by flow cytometry and in in-vitro stimulation protocols now allow reproducible detection of IL-10 expression by B cells after only five hours - greatly improving accuracy of phenotyping [83]. After such stimulation, IL-10+ B cells typically represent $\sim 1 \%$ of total splenic B cells, although they are enriched (e.g., 10$25 \%$ ) in certain B cell subpopulations. Yet, it remains uncertain how stimulation of B cells with phorbol esters, ionomycin, and LPS relate to what actually occurs in vivo. Moreover, the number of B cells expressing IL-10 increases 3-5 fold if cells are first cultured for 48 hours with CD40ligation followed by the above cocktail for five hours [10]. It has been proposed that cells capable of IL-10 expression in five hours are functional Bregs, whereas those that become IL10-competent after 48 hours are Breg progenitors [10]. However, direct evidence that Bregs really belong to a specific 
lineage is lacking because there is no definitive marker or transcriptional signature. Rather, IL-10 expression may represent a state activation that is directed or stochastic.

Using such stimulatory conditions, various B cell subsets were shown to be enriched for IL-10 and such subsets exhibit Breg activity upon adoptive transfer. Thus, splenic marginal zone (MZ) [88-90], less-mature MZ-precursor (MZ-P) or Transitional 2 (T2) [79, 81, 91], follicular (FO) [81, 90, 92] $\mathrm{B}$ cells, and those expressing a CD1dhiCD5+ phenotype that partially overlaps with MZ and B1 B cells [83] have all been described as regulatory. However, these appear to contain the highest proportion of IL-10+ B cells in particular models, rather than representing a true "Breg phenotype". This is based in the following two issues. First, despite being enriched, IL-10+ B cells remain a minority of the B cells in these subsets. Those subsets with the highest frequency of IL$10+$ cells (and likely, with the lowest frequency of proinflammatory B cells) will appear to be regulatory compared to other subsets where the frequency of IL-10+ B cells is lower. (The ratio of pro and anti-inflammatory B cells in a given subset is particularly evident in humans, as detailed below). Second, many of these B cell subsets are small and while enriched, they contain only a fraction of total IL-10 expressing B cells. For example, a B cell subset may be highly enriched for IL- $10+\mathrm{B}$ cells $(25 \%)$ but may comprise only $2 \%$ of the total B cell population. If $1 \%$ of the remaining $98 \%$ of the $\mathrm{B}$ cell population express IL-10, this will contain twice as many IL-10+ B cells as the smaller population. While the larger subset may not exhibit regulatory activity due to the low frequency of IL-10+ B cells, referring to the small, enriched subset as "the Breg population" is misleading. At present, it is really not known whether IL-10+ B cells belonging to various B cell subpopulations have similar or distinct activities. Nor is it known whether IL-10- cells within certain subpopulations can contribute to the regulatory activity of the IL-10+ subpopulation. Recent advances (such as selection of TIM-1+ B cells discussed below, or in-vitro expansion) may allow enrichment of IL-10+ B cells belonging to different subsets to be compared directly. As an additional source of confusion, the small CD1dhiCD5+ population was initially dubbed "B10" because it was highly enriched for IL-10 expression. While this subset actually contains only $20-25 \%$ of IL-10+ B cells [18••], others have used "B10" to describe any IL-10+ B cells.

TIM-1 is an Inclusive Marker for Bregs That Have Activity in Allograft Models:

TIM-1 is a member of the T immunoglobulin and mucin domain family of costimulatory molecules. We discovered that TIM- 1 is constitutively expressed on $6-8 \%$ of B cells, and increases after immunization [18••]. Importantly, TIM-1+ $B$ cells are 10-30 fold enriched for cells expressing IL-10 compared to their TIM-1- counterparts, in every B cell subset examined (including CD1dHiCD5+ and FO B cells). Thus, as a single marker, TIM-1 identifies $\sim 70 \%$ of all IL-10+ B cells, making it the most inclusive marker of IL-10 expressing B cells $[18 \bullet \bullet]$.

The regulatory role of TIM-1+ B cells was demonstrated by showing that TIM-1+, but not TIM-1-, B cells from alloimmunized mice could transfer long-term islet allograft survival into otherwise untreated B-deficient recipients. IL-10 expression was essential for graft prolongation. Since TIM-1+ B cells are enriched for IL-10+ cells from various B cell subpopulations, our findings suggest that IL-10+ cells in different B cell subsets are likely to exhibit Breg activity, and TIM-1 may provide sufficient enrichment to directly test this.

We found that TIM-1+ Bregs transferred from naïve mice were ineffective at prolonging allograft survival, while Bregs transferred from alloimmunized hosts prolonged allograft survival in an antigen-specific manner [18••]. This is in agreement with the general finding that Bregs transferred from naïve mice are ineffective, or less potent than those from immunized mice $[18 \bullet \bullet, 81,83]$ and suggests that control of aggressive immune responses requires prior expansion of antigen-specific Breg clones [18••]. In collagen-induced arthritis, Bregs were less potent when transferred from mice during the acute phase of the disease, and more potent after disease resolution [81]. Nonetheless, TIM-1+ B cells from acutely allo-immunized mice could transfer tolerance to Bdeficient allograft recipients $[18 \bullet \bullet]$. Lee et al. extended these findings by showing that whole B cells from tolerant mice (treated with anti-TIM-1 and anti-CD45RB) could transfer tolerance to wild-type allograft recipients. Whether cells from tolerant mice are more potent than those from acutely immunized mice was not examined. An unresolved question is whether B cells from mice that are tolerant (or after disease resolution), express more IL-10+ Bregs, or have less proinflammatory B cells mixed into the transferred population.

\section{The Role of TIM-1 and Bregs in Allograft Models}

The studies above show that TIM-1 is an inclusive marker for IL-10-expressing B cells that can transfer allospecific tolerance. To gain further insight into the role of Bregs in allograft models we examined a tolerogenic low-affinity anti-TIM-1 mAb (RMT1-10) [93, 94]. This mAb had been previously shown to prolong allograft survival (and inhibit EAE) by skewing the immune response from a Th1 to a Th2 response and promoting Tregs $[93,94]$. We found that in the absence of $\mathrm{B}$ cells, this same anti-TIM-1 mAb actually accelerated rejection, suggesting a costimulatory effect on $\mathrm{T}$ cells similar to higher-affinity anti-TIM-1 mAbs [94, 95]. Prolonged graft survival could be restored by reconstituting anti-Tim-1-treated B-deficient mice with wild-type but not IL-10-deficient B cells. Moreover, the salutary CD4 responses induced by anti- 
TIM-1 were all B cell-dependent. Thus, in the absence of B cells, anti-TIM-1 enhanced IFN $\gamma$ expression and no longer increased IL-4, IL-10, or Treg number. Breg transfer had similar effects on CD4 cells as anti-TIM-1 treatment. These data suggest that in allograft settings, Bregs promote less injurious Th2 responses, reduce Th1 responses, and enhance Tregs. Bregs have been shown to enhance Tregs in several studies [18••, 96, 97], likely through their expression of IL-10 and TGF $\beta$ [98-101]. However, Breg activity was found to be either distinct from, or independent of, Tregs in collagen-induced arthritis and EAE models [81, 102]. In allograft models, it is uncertain whether the reported requirement for Tregs in the presence of Bregs is due to independent or interdependent effects [103].

Given that anti-TIM-1 only prolongs allograft survival in the presence of B cells and that TIM-1 is expressed by Bregs, we examined the effect of this $\mathrm{mAb}$ on $\mathrm{B}$ cells. We found that anti-TIM-1 enhances TIM-1 and IL-10 expression, giving rise to a five-fold induction of IL-10+ B cells [18••]. Taken together, our data indicate for the first time that Bregs can be directly targeted for expansion in vivo, and moreover, that anti-TIM-1 promotes tolerance through a Breg-dependent mechanism.

\section{Breg Expansion for Therapeutic Purposes:}

The finding that anti-TIM-1 can induce Bregs indicates that TIM-1 is more than a marker for Bregs and TIM-1 signaling is involved in Breg induction. In this regard, it was previously demonstrated that administration of apoptotic cells induces B cell IL-10 expression, and treated mice are resistant to EAE and collagen-induced arthritis [90, 104•]. This appears to involve antigen-binding by self-reactive BCRs (enriched on natural IgM-expressing MZ B cells) and TLR-9 signals. However, TIM-1 is expressed by B cells enriched for IL-10 expression and is a known phosphatidylserine (PS) receptor [105]. This raises the question as to whether TIM-1 is also involved in AC binding and IL-10 production by B cells. It should be noted that anti-CD40 stimulation of B cells ex vivo has been shown to enhance activity of transferred Bregs [91]. Finally, Tedder and colleagues recently shown that Bregs can be dramatically expanded in vitro through CD40 ligation and provision of IL-21 [106••]. Thus, there are various ways that Bregs might be manipulated for therapeutic applications.

\section{The Broader Role of Bregs in Allograft Models:}

The loss of ability of anti-TIM-1 to prolong allograft survival in the absence of $\mathrm{B}$ cells is reminiscent of the previously unexplained B cell-dependence of anti-CD45RB, a therapy that we previously showed can induce Tregs [107-109]. Preliminary findings of ours and others suggest that anti-CD154+ DST and anti-TIM-4 also require B cells for allograft tolerance ([110] and DR unpublished data). While anti-TIM-1 is unique in that allograft survival primarily occurs through Breg induction, these studies suggest that Bregs may be essential to establish a set-point for allograft tolerance induced by various agents that do not necessarily directly target Bregs. In this regard, the absence of Bregs may raise the threshold for tolerance by augmenting immune responses, much in the same way as Tregs.

A new Subset of Regulatory B Cells: IL-35 Producing Plasmablasts:

A recent report reveals that $\mathrm{B}$ cells can also secrete the suppressive cytokine IL-35, and mice with B cells deficient in IL-35 are more susceptible to EAE but exhibit enhanced resistance to salmonella compared to those with wild-type B cells [111••]. IL-35 and IL-10 were expressed by distinct B cells - suggesting that both may operate in different fashions to suppress EAE. Surprisingly, most IL-10 and IL-35 expression in mice after salmonella infection or induction of EAE came from $\mathrm{IgM}+\mathrm{CD} 138+$ plasmablasts, challenging the dogma that plasma cells' sole function is to produce antibodies and also raising questions about the degree to which Bregs exert their activity as B cells versus plasma cells. However, it is uncertain whether immature B cells (or transitional or MZ precursors), found to transfer Breg activity in many models, must fully mature into plasma cells before exertive suppressive activity.

\section{Bregs in Humans}

Role of B Cells in Allograft Survival and Tolerance:

B cell depletion with Rituximab is effective in treating several human autoimmune diseases by disrupting proinflammatory and costimulatory functions of B cells. However, B cell depletion can also lead to exacerbations or de-novo manifestations of autoimmune disease (reviewed in [112, 113]). Importantly, at least in certain settings, B cell depletion prior to transplantation may markedly increase the incidence of acute cellular rejection [114]. These studies lend support to the presence of Bregs and to the importance of trying to better identify inflammatory and regulatory subsets for more selective targeting. However, recent data detailed below suggest that this may not be straightforward.

Identification of Human Bregs:

Recent studies provide new insight into human regulatory B cell phenotype and IL-10 expression. As in mice, IL-10 protein expression is not observed in human B cells unless they are activated in vitro $[115,116 \cdot, 117]$. 
In humans, transitional B cells (TrB; $\mathrm{CD} 24^{\mathrm{hi}} \mathrm{CD} 38^{\mathrm{hi}}$ ) from peripheral blood of healthy volunteers were shown to be enriched for IL-10 expression and capable of suppressing inflammatory cytokine expression by autologous $\mathrm{CD} 4^{+} \mathrm{T}$ cells in vitro [117]. In this regard, tolerant renal allograft patients were shown to express higher numbers of $B$ cells, $\operatorname{TrB}$, and more IL-10+ TrB than immunosuppressed patients with stable renal allograft function [118, 119]. Moreover, $\operatorname{TrB}$ cells from SLE patients were shown to express less IL-10 in response to CD40 ligation than those from healthy controls and lacked invitro regulatory activity [117]. In contrast, Iwata et al. showed that IL-10 is highest in human $\mathrm{CD} 24^{\mathrm{hi}} \mathrm{CD} 27^{+}$memory B cells (MemB) and that IL-10 expression in patients with various autoimmune diseases including SLE and RA is actually higher than in healthy controls [116•]. Thus, there is a discrepancy in the current literature on the identity and role of human Bregs.

To readdress this issue, we directly compared B cell subpopulations from healthy controls. Given that cells within these subpopulations are not uniform, and IL-10 is expressed by only a fraction of these $\mathrm{B}$ cells, we asked whether the concomitant expression of pro-inflammatory cytokines influenced the regulatory activity observed. We examined B cell expression of TNF $\alpha$ based on its importance in both antibodymediated responses in mice and in promoting $\mathrm{T}$ cell responsiveness in MS [13, 120••, 121]. After stimulation (CPG and CD40L) for $48 \mathrm{~h}$, we found that IL-10 expression by B cells in isolated healthy volunteers (HV) was similarly enriched amongst Memory (MemB), and Transitional B (TrB) compared to naïve B cells $[120 \bullet \cdot$. However, a significant fraction of B cells within each subset co-expressed TNF $\alpha$, and in fact, many B cells simultaneously expressed both cytokines. Compared to MemB, TrB expressed similar IL-10, but much lower $\mathrm{TNF} \alpha$, resulting in a higher IL-10:TNF $\alpha$ ratio ( $\sim 1.5$ vs. 0.6$)$. Similar results were obtained when B cells were sorted into subsets prior to in-vitro stimulation. The IL-10:TNF $\alpha$ ratio correlated with potent suppression of Th1 cytokines in vitro by $\operatorname{TrB}$, but not MemB or naïve B subsets. Neutralization of IL-10 blocked TrB regulatory function, while neutralization of $\mathrm{TNF} \alpha$ uncovered regulatory function of MemB, showing that these cytokines are involved in in-vitro suppressive activity.

We then examined the utility of the TrB IL-10/TNF- $\alpha$ ratio in a cross-sectional study of 88 renal transplant recipients 2-15 years post-transplant ${ }^{120}$. Patients with stable allograft function and those with graft dysfunction but no rejection had $\mathrm{TrB}$ IL-10:TNF $\alpha$ ratios comparable to HV. In contrast, TrB from patients with graft dysfunction and rejection were not only reduced in number, but exhibited a more inflammatory cytokine profile with a significant fall in IL-10:TNF $\alpha$ ratio and they lost their in-vitro regulatory activity. Neither TrB IL-10 alone, nor IL-10:TNF $\alpha$ ratio of total B cells correlated with rejection. Thus, human $\mathrm{TrB}$ may exhibit more suppressive or inflammatory activity depending on the subject's immunological status.
Importantly, the TrB IL-10/TNF- $\alpha$ ratio at the time of forcause biopsy was a predictor of graft outcomes over a threeyear follow-up ${ }^{120}$. Amongst 47 patients with for-cause biopsies, those with a high ratio (above the overall group mean) had stable graft function, whereas $40 \%$ of those with low ratios had a two-fold decrease in GFR or graft loss over three years. Amongst the relatively small number with rejection, there was a strong trend toward poor outcome that did not quite reach statistical significance $(p=0.056)$. Of note, in this study, rejection was primarily due to chronic, antibodymediated rejection (AMR), while $25 \%$ had superimposed, acute cellular rejection (ACR).

These findings suggest that standard human B cell subsets are insufficient in separating potentially suppressive and inflammatory B cells. Additionally, current in-vitro simulation protocols induce both pro- and anti-inflammatory cytokines by the same cells. The IL-10/TNF- $\alpha$ ratio not only correlated with in-vitro suppressive function, but both the ratio and invitro activity were altered in the presence of an active immune (rejection) response. Taken together, our results imply that the cytokine ratio within the TrB subset is a much more accurate way to gauge suppressive versus effector function. This is important because this ratio changes in the presence of immunological activity. While these findings clearly require confirmation in a larger cohort, they suggest that at the time of biopsy, the IL-10/TNF- $\alpha$ ratio may be predictive of outcome. In turn, these findings amplify the notion that both B cell inflammatory and anti-inflammatory cytokines play an important role in immune response. It remains to be determined whether such changes can be used prospectively to guide therapy for patients at risk.

\section{Conclusion}

It is now evident that in addition to their role in humoral immunity, B cells play an important role in modulating $\mathrm{T}$ cell-mediated immune responses. Studies in mice with specific $\mathrm{B}$ cell defects demonstrate key roles for B cells in enhancing effector $\mathrm{T}$ cell and memory $\mathrm{T}$ cell responses on the one hand, and inhibiting effector $\mathrm{T}$ cell responses and promoting tolerance on the other. In allograft models, effector B cells play an important role as APCs that promote T cell activation and chronic rejection. Production of the inflammatory cytokine, $\mathrm{TNF} \alpha$, by B cells also augments production of antibodies by $B$ cells and may play a role in promoting humoral immunity in humans. Alternatively, regulatory B cells help establish a setpoint for allograft tolerance in murine models and may promote immune quiescence in both tolerant and immunosuppressed human renal allograft recipients. While targeting CD20+ B cells is of unclear benefit in treating antibodymediated rejection, this approach inhibits ongoing $\mathrm{T}$ cell 
responses in autoimmune disease, and could have a role in treating acute rejection and/or inhibiting chronic rejection. On the other hand, non-selective depletion of all $\mathrm{B}$ cells has the potential to dysregulate the immune system and even precipitate acute rejection. If confirmed, recent findings that plasmablasts may be potent immune regulators only complicates new approaches aimed at specifically targeting these antibodyproducing cells. Thus, in the allograft setting we need to identify approaches to selectively deplete or inhibit effector B cells while enhancing regulatory B cells. The finding that the same B cells can secrete both regulatory and inflammatory cytokines further complicates this task. If these in-vitro findings correspond to their capacity in vivo, simple depletion based on phenotype may not be possible. Clearly, we have much to learn about the immunobiology of effector and regulatory B cells, starting with more accurate identification, understanding their plasticity. In this regard little is known about where or how these cells function in vivo. Understanding this, may help us gain insight into selective inhibition or enhancement of their function. In the meantime, if the utility of the ratio of B cell inflammatory to inhibitory cytokines as a marker for subsequent clinical course is confirmed, it may identify patients whose clinical course can be modified by more intensive immunosuppression.

Acknowledgments This work was supported by grants NIH AI079177 (GC), ROTRF 978906253 (GC), and NIH AI097361 (DR).

\section{Compliance with Ethics Guidelines}

Conflict of Interest Geetha Chalasani and David Rothstein declare that they have no conflicts of interest.

Human and Animal Rights and Informed Consent This article does not contain any studies with human or animal subjects performed by any of the authors.

\section{References}

Papers of particular interest, published recently, have been highlighted as:

- Of importance

•- Of major importance

1. Matsushita T, Yanaba K, Bouaziz JD, Fujimoto M, Tedder TF. Regulatory B cells inhibit EAE initiation in mice while other B cells promote disease progression. J Clin Invest. 2008;118:3420-30.

2. Kissmeyer-Nielsen F, Olsen S, Petersen V, Fjeldborg O. Hyperacute rejection of kidney allografts associated with with pre-existing humoral antibodies against donor cells. Lancet. 1966;2:662-5.

3. Jeannet M, Pinn VW, Flax MH, Winn HJ, Russell PS. Humoral antibodies in renal allotransplantation in man. $\mathrm{N}$ Engl $\mathrm{J}$ Med. 1970;282:111-7.

4. Lachmann $\mathrm{N}$ et al. Anti-human leukocyte antigen and donor-specific antibodies detected by luminex posttransplant serve as biomarkers for chronic rejection of renal allografts. Transplantation. 2009;87:1505-13.
5. Wiebe $\mathrm{C}$ et al. Evolution and clinical pathologic correlations of de novo donor-specific HLA antibody post kidney transplant. Am J Transplant. 2012;12:1157-67.

6. Loupy A et al. Complement-binding anti-HLA antibodies and kidney-allograft survival. N Engl J Med. 2013;369:1215-26.

7. Janeway Jr CA, Ron J, Katz ME. The B cell is the initiating antigen-presenting cell in peripheral lymph nodes. J Immunol. 1987;138:1051-5.

8. Ron Y, Sprent J. T cell priming in vivo: a major role for B cells in presenting antigen to $\mathrm{T}$ cells in lymph nodes. J Immunol. 1987;138:2848-56.

9. Harris DP et al. Reciprocal regulation of polarized cytokine production by effector B and T cells. Nat Immunol. 2000;1:475-82.

10. Dilillo DJ, Matsushita T, Tedder TF. B10 cells and regulatory B cells balance immune responses during inflammation, autoimmunity, and cancer. Ann N Y Acad Sci. 2010;1183:38-57.

11. Lund FE, Randall TD. Effector and regulatory B cells: modulators of CD4+ T cell immunity. Nat Rev Immunol. 2010;10:236-47.

12. Fillatreau S, Sweenie CH, McGeachy MJ, Gray D, Anderton SM. B cells regulate autoimmunity by provision of IL-10. Nat Immunol. 2002;3:944-50.

13. Wojciechowski $\mathrm{W}$ et al. Cytokine-producing effector B cells regulate type 2 immunity to $H$. polygyrus. Immunity. 2009;30:421-33.

14. • Barr TA et al. B cell depletion therapy ameliorates autoimmune disease through ablation of IL-6-producing B cells. J Exp Med. 2012;209:1001-10. This study demonstrates that IL-6 expressed by $B$ cells is a major mediator of autoimmune disease mediated by $T$ cells, including EAE (mice) and MS (humans).

15. Cross AH, Stark JL, Lauber J, Ramsbottom MJ, Lyons JA. Rituximab reduces B cells and T cells in cerebrospinal fluid of multiple sclerosis patients. J Neuroimmunol. 2006;180:63-70.

16. Edwards JC et al. Efficacy of B-cell-targeted therapy with rituximab in patients with rheumatoid arthritis. $N$ Engl $\mathrm{J}$ Med. 2004;350:2572-81.

17. Bouaziz JD et al. Therapeutic B cell depletion impairs adaptive and autoreactive CD4+ T cell activation in mice. Proc Natl Acad Sci U S A. 2007;104:20878-83.

18.• Ding $\mathrm{Q}$ et al. This study indentifies TIM-1 as an inclusive marker for Bregs and as a target for Breg induction, and demonstrates that Bregs can promote tolerance in allograft models. J Clin Invest. 2011;121:3645-56. This study indentifies TIM-1 as an inclusive marker for Bregs and as a target for Breg induction, and demonstrates that Bregs can promote tolerance in allograft models.

19. Fiorina $\mathrm{P}$ et al. Targeting CD22 reprograms B-cells and reverses autoimmune diabetes. Diabetes. 2008;57:3013-24.

20. Hu CY et al. Treatment with CD20-specific antibody prevents and reverses autoimmune diabetes in mice. J Clin Invest. 2007;117: 3857-67.

21. Mauri C, Gray D, Mushtaq N, Londei M. Prevention of arthritis by interleukin 10-producing B cells. J Exp Med. 2003;197:489-501.

22. Martin F, Kearney J. B-cell subsets and the mature preimmune repertoire. Marginal zone and B1 B cells as part of a "natural immune memory". Immunol Rev. 2000;175:70-9.

23. Bayry $\mathrm{J}$ et al. Modulation of dendritic cell maturation and function by B lymphocytes. J Immunol. 2005; 175:15-20.

24. Boes M. Role of natural and immune IgM antibodies in immune responses. Mol Immunol. 2000;37:1141-9.

25. Mitchison NA. T-cell-B-cell cooperation. Nat Rev Immunol. 2004;4:308-12.

26. Lau CM et al. RNA-associated autoantigens activate B cells by combined B cell antigen receptor/Toll-like receptor 7 engagement. J Exp Med. 2005;202:1171-7.

27. Rifkin IR, Leadbetter EA, Busconi L, Viglianti G, MarshakRothstein A. Toll-like receptors, endogenous ligands, and systemic autoimmune disease. Immunol Rev. 2005;204:27-42. 
28. Leadbetter EA et al. Chromatin-IgG complexes activate B cells by dual engagement of $\operatorname{IgM}$ and Toll-like receptors. Nature. 2002;416:603-7.

29. Krieg AM et al. CpG motifs in bacterial DNA trigger direct B-cell activation. Nature. 1995;374:546-9.

30. Akiba $\mathrm{H}$ et al. CD28-independent co-stimulation of $\mathrm{T}$ cells by OX40 ligand and CD70 on activated B cells. J Immunol. 1999;162:7058-65.

31. Barr TA, Brown S, Ryan G, Zhao J, Gray D. TLR-mediated stimulation of APC: Distinct cytokine responses of B cells and dendritic cells. Eur J Immunol. 2007;37:3040-53.

32. Uehara S, Chase CM, Colvin RB, Russell PS, Madsen JC. Further evidence that NK cells may contribute to the development of cardiac allograft vasculopathy. Transplant Proc. 2005;37:70-1.

33. Hirohashi $\mathrm{T}$ et al. A novel pathway of chronic allograft rejection mediated by NK cells and alloantibody. Am J Transplant. 2012;12:313-21.

34. Jindra PT et al. Anti-MHC class I antibody activation of proliferation and survival signaling in murine cardiac allografts. $\mathrm{J}$ Immunol. 2008;180:2214-24.

35. Burns AM, Chong AS. Alloantibodies prevent the induction of transplantation tolerance by enhancing alloreactive $\mathrm{T}$ cell priming. J Immunol. 2011;186:214-21.

36. Snider D, Segal D. Efficiency of antigen presentation after antigen targeting to surface IgD, IgM, MHC, Fc $\gamma$ RII, and B220 molecules on murine splenic B cells. J Immunol. 1989;143:59-65.

37. Lanzavecchia A. Antigen-specific interaction between $\mathrm{T}$ and $\mathrm{B}$ cells. Nature. 1985;314:537-9.

38. Phan T, Grigorova I, Okada T, Cyster J. Subcapsular encounter and complement-dependent transport of immune complexes by lymph node B cells. Nat Immunol. 2007;8:992-1000.

39. Phan TG, Green JA, Gray EE, Xu Y, Cyster JG. Immune complex relay by subcapsular sinus macrophages and noncognate B cells drives antibody affinity maturation. Nat Immunol. 2009;10:78693.

40. Kurt-Jones $\mathrm{E}$ et al. The role of antigen-presenting B cells in $\mathrm{T}$ cell priming in vivo: Studies of B cell-deficient mice. J Immunol. 1988;140:3773-8.

41. Fuchs EJ, Matzinger P. B cells turn off virgin but not memory T cells. Science. 1992;258:1156-9.

42. Fu YX, Huang G, Wang Y, Chaplin DD. B lymphocytes induce the formation of follicular dendritic cell clusters in a lymphotoxin alpha-dependent fashion. J Exp Med. 1998;187:1009-18.

43. Nolte MA et al. B cells are crucial for both development and maintenance of the splenic marginal zone. J Immunol. 2004;172: 3620-7.

44. Ngo VN, Cornall RJ, Cyster JG. Splenic T zone development is B cell dependent. J Exp Med. 2001;194:1649-60.

45. Nozaki T, Rosenblum JM, Ishii D, Tanabe K, Fairchild RL. CD4 T cell-mediated rejection of cardiac allografts in B cell-deficient mice. J Immunol. 2008;181:5257-63.

46. Shen $\mathrm{H}$ et al. A specific role for $\mathrm{B}$ cells in the geneartion of CD8 $\mathrm{T}$ cell memory by recombinant Listeria monocytogenes. J Immunol. 2003;170:1443-51.

47. Lund FE, Schuer K, Hollifield M, Randall TD, Garvy BA. Clearance of Pneumocystis carinii in mice is dependant on B cells but not on P.carinii-specific antibody. J Immunol. 2003;171:1423-30.

48. Lund $\mathrm{F}$ et al. B cells are required for generation of protective effector and memory CD4 cells in response to Pneumocystis lung infection. J Immunol. 2006;176:6147-54.

49. Crawford A, MacLeod M, Schumacher T, Corlett L, Gray D. Primary $\mathrm{T}$ cell expansion and differentiation in vivo requires antigen presentation by B cells. J Immunol. 2006;176:3498-506.

50. Barr TA, Brown S, Mastroeni P, Gray D. TLR and B cell receptor signals to B cells differentially program primary and memory Th1 responses to Salmonella enterica. J Immunol. 2010;185:2783-9.
51. Whitmire $\mathrm{J}$ et al. Requirement of $\mathrm{B}$ cells for generating CD4+ $\mathrm{T}$ cell memory. J Immunol. 2009;182:1868-76.

52. Russell PS, Chase CM, Winn HJ, Colvin RB. Coronary atherosclerosis in transplanted mouse hearts II Importance of humoral immunity. J Immunol. 1994;152:5135-41.

53. Levesque $\mathrm{V}$ et al. B-cell-dependent memory $\mathrm{T}$ cells impede nonmyeloablative mixed chimerism induction in presensitized mice. Am J Transplant. 2011;11:2322-31.

54. Ng Y, Oberbarnscheidt M, Chandramoorthy H, Hoffman R, Chalasani G. B cells help alloreactive T cells differentiate into memory T cells. Am J Transplant. 2010;10:1970-80.

55. Noorchashm $\mathrm{H}$ et al. B cell-mediated antigen presentation is required for the pathogenesis of acute cardiac allograft rejection. J Immunol. 2006;177:7715-22.

56. Zeng Q et al. B cells mediate chronic allograft rejection independently of antibody production. J Clin Invest. 2014;124:1052-6. This study establishes that secreted antibodies are not essential, and antibody-independent functions of B cells are sufficient for development of chronic rejection. Cognate and noncognate functions of $B$ cells contribute to chronic rejection by supporting $T$ cell responses through antigen presentation and maintenance of lymphoid architecture.

57. Kitamura D, Roes J, Kuhn R, Rajewsky K. A B cell-deficient mouse by targeted disruption of the membrane exon of the immunoglobulin $\mu$ chain gene. Nature. 1991;350:423-6.

58. Kumazaki K et al. AID-/-mus-/- mice are agammaglobulinemic and fail to maintain B220-CD138+ plasma cells. J Immunol. 2007;178:2192-203.

59. O'Neill SK et al. Expression of CD80/86 on B cells is essential for autoreactive $\mathrm{T}$ cell activation and the development of arthritis. $\mathrm{J}$ Immunol. 2007;179:5109-16.

60. Viau M, Zouali M. B-lymphocytes, innate immunity, and autoimmunity. Clin Immunol. 2005;11:17-26.

61. Attanavanich K, Kearney JF. Marginal zone, but not follicular B cells, are potent activators of naive CD4 $\mathrm{T}$ cells. J Immunol. 2004; 172:803-11.

62. Oliver AM, Martin F, Kearney JF. IgMhighCD21high lymphocytes enriched in the splenic marginal zone generate effector cells more rapidly than the bulk of follicular B cells. J Immunol. 1999;162:7198-207.

63. McDonald KG, McDonough JS, Newberry RD. Adaptive immune responses are dispensable for isolated lymphoid follicle formation: antigen-naive, lymphotoxin-sufficient B lymphocytes drive the formation of mature isolated lymphoid follicles. J Immunol. 2005;174:5720-8.

64. Baddoura $\mathrm{F}$ et al. Lymphoid neogenesis in murine cardiac allografts undergoing chronic rejection. Am J Transplant. 2005;5: 510-6.

65. Nasr I et al. Tertiary lymphoid tissues generate effector and memory $\mathrm{T}$ cells that lead to allograft rejection. Am J Transplant. 2007;7:1071-9.

66. Thaunat $\mathrm{O}$ et al. Lymphoid neogenesis in chronic rejection: evidence for a local humoral alloimmune response. Proc Natl Acad Sci U S A. 2005;102:14723-8.

67. Motallebzadeh $\mathrm{R}$ et al. Blocking lymphotoxin signaling abrogates the development of ectopic lymphoid tissue within cardiac allografts and inhibits effector antibody responses. FASEB J. 2012;26: 51-62.

68. Linton $\mathrm{P}$ et al. Costimulation via OX40L expressed by B cells is sufficient to determine the extent of primary CD4 cell expansion and Th2 cytokine secretion in vivo. J Exp Med. 2003;197:875-83.

69. Watts TH. TNF/TNFR Family members in costimulation of T cell responses. Annu Rev Immunol. 2005;23:23-68.

$70 . \bullet \mathrm{Xu} \mathrm{H}$ et al. Follicular T-helper cell recruitment governed by bystander B cells and ICOS-driven motility. Nature. 2013;496: 523-7. This study identifies a novel function of non- 
cognate bystander B cells in providing ICOSL-mediated costimulation to TFH precursors essential for their motility into $B$ cell follicles to compete TFH differentiation.

71. Weinstein JS et al. B cells in T follicular helper cell development and function: separable roles in delivery of ICOS ligand and antigen. J Immunol. 2014;192:3166-79. This study dissects the requirement of antigen presentation $v$ s. ICOSL costimulation by $B$ cells in TFH development and function. When cognate B cells are abundant and present specific antigens to T cells, TFH differentiation and $G C$ responses can occur independently of ICOSL costimulation by B cells.

72. Hamel KM, Cao Y, Olalekan SA, Finnegan A. B cell-specific expression of inducible costimulator ligand is necessary for the induction of arthritis in mice. Arthr Rheumatol. 2014;66:60-7. This study shows that in the absence of ICOSL on B cells, proteoglycan-induced arthritis is attenuated and GCs,TFH and IFN $\gamma, I L-17, I L-4$ and IL-21 cytokines are reduced.

73. Menard LC et al. B cells amplify IFN-gamma production by T cells via a TNF-alpha-mediated mechanism. J Immunol. 2007;179:4857-66.

74.• Rauch PJ et al. Innate response activator B cells protect against microbial sepsis. Science. 2012;335:597-601. This study identifies a novel B cell population that produced GM-CSF in response to LPS and polymicrobial sepsis. GM-CSF production by B cells supports neutrophil functions in mediating bacterial clearance and reduce mortality in sepsis.

75. Moulin V et al. B lymphocytes regulate dendritic cell (DC) function in vivo: increased interleukin 12 production by DCs from B cell-deficient mice results in T helper cell type 1 deviation. J Exp Med. 2000;192:475-82.

$76 . \bullet$ Zouggari $\mathrm{Y}$ et al. B lymphocytes trigger monocyte mobilization and impair heart function after acute myocardial infarction. Nat Med. 2013;19:1273-80. This study identifies a novel role for B cells in myocardial infarction as mediators of inflammatory monocyte recruitment into the myocardium by producing $\mathrm{Ccl}$. B cell depletion after myocardial infarction led to decreased inflammatory monocytes in myocardium, better cardiac function, and reduced systemic inflammation.

77. Neta R, Salvin SB. Specific suppression of delayed hypersensitivity: the possible presence of a suppressor B cell in the regulation of delayed hypersensitivity. J Immunol. 1974;113:1716-25.

78. Mizoguchi A, Mizoguchi E, Smith RN, Preffer FI, Bhan AK. Suppressive role of B cells in chronic colitis of T cell receptor alpha mutant mice. J Exp Med. 1997;186:1749-56.

79. Mizoguchi A, Mizoguchi E, Takedatsu H, Blumberg RS, Bhan AK. Chronic intestinal inflammatory condition generates IL-10producing regulatory $\mathrm{B}$ cell subset characterized by CD1d upregulation. Immunity. 2002;16:219-30.

80. Wolf SD, Dittel BN, Hardardottir F, Janeway Jr CA. Experimental autoimmune encephalomyelitis induction in genetically B celldeficient mice. J Exp Med. 1996;184:2271-8.

81. Evans JG et al. Novel suppressive function of transitional 2 B cells in experimental arthritis. J Immunol. 2007;178: 7868-78.

82. Watanabe R et al. CD19 expression in B cells is important for suppression of contact hypersensitivity. Am J Pathol. 2007;171: 560-70.

83. Yanaba $\mathrm{K}$ et al. A regulatory $\mathrm{B}$ cell subset with a unique CD1dhiCD5+ phenotype controls T cell-dependent inflammatory responses. Immunity. 2008;28:639-50.

84. Hussain S, Delovitch TL. Intravenous transfusion of BCRactivated B cells protects NOD mice from type 1 diabetes in an IL-10-dependent manner. J Immunol. 2007;179:7225-32.

85. Rowe $\mathrm{V}$ et al. Host $\mathrm{B}$ cells produce IL-10 following TBI and attenuate acute GVHD after allogeneic bone marrow transplantation. Blood. 2006;108:2485-92.
86. Mauri C, Ehrenstein MR. The 'short' history of regulatory B cells. Trends Immunol. 2008;29:34-40.

87. Haas KM et al. Protective and pathogenic roles for B cells during systemic autoimmunity in NZB/W F1 mice. J Immunol. 2010;184:4789-800.

88. Brummel R, Lenert P. Activation of marginal zone B cells from lupus mice with type A(D) CpG-oligodeoxynucleotides. J Immunol. 2005;174:2429-34.

89. Lenert P, Brummel R, Field EH, Ashman RF. TLR-9 activation of marginal zone $\mathrm{B}$ cells in lupus mice regulates immunity through increased IL-10 production. J Clin Immunol. 2005;25:29-40.

90. Gray M, Miles K, Salter D, Gray D, Savill J. Apoptotic cells protect mice from autoimmune inflammation by the induction of regulatory B cells. Proc Natl Acad Sci U S A. 2007;104:14080-5.

91. Blair PA et al. Selective targeting of B cells with agonistic antiCD40 is an efficacious strategy for the generation of induced regulatory T2-like B cells and for the suppression of lupus in MRL/lpr mice. J Immunol. 2009;182:3492-502.

92. Miyazaki D, Kuo CH, Tominaga T, Inoue Y, Ono SJ. Regulatory function of CpG-activated B cells in late-phase experimental allergic conjunctivitis. Invest Ophthalmol Vis Sci. 2009;50: 1626-35.

93. Ueno $\mathrm{T}$ et al. The emerging role of T cell Ig mucin 1 in alloimmune responses in an experimental mouse transplant model. J Clin Invest. 2008;118:742-51.

94. Xiao $\mathrm{S}$ et al. Differential engagement of Tim-1 during activation can positively or negatively costimulate $\mathrm{T}$ cell expansion and effector function. J Exp Med. 2007;204:1691-702.

95. Degauque $\mathrm{N}$ et al. Immunostimulatory Tim-1-specific antibody deprograms Tregs and prevents transplant tolerance in mice. J Clin Invest. 2008;118:735-41.

96. Mann MK, Maresz K, Shriver LP, Tan Y, Dittel BN. B cell regulation of $\mathrm{CD} 4+\mathrm{CD} 25+\mathrm{T}$ regulatory cells and $\mathrm{IL}-10$ via $\mathrm{B} 7$ is essential for recovery from experimental autoimmune encephalomyelitis. J Immunol. 2007;178:3447-56.

97. Amu S et al. Regulatory B cells prevent and reverse allergic airway inflammation via FoxP3-positive T regulatory cells in a murine model. J Allergy Clin Immunol. 2010;125:1114-24.

98. Singh A et al. Regulatory role of B cells in a murine model of allergic airway disease. J Immunol. 2008;180:7318-26.

99. Tian J et al. Lipopolysaccharide-activated B cells down-regulate Th1 immunity and prevent autoimmune diabetes in nonobese diabetic mice. J Immunol. 2001;167:1081-9.

100. Carter NA, Rosser EC, Mauri C. Interleukin-10 produced by B cells is crucial for the suppression of Th17/Th1 responses, induction of T regulatory type 1 cells and reduction of collagen-induced arthritis. Arthritis Res Ther. 2012;14:R32.

101. Moritoki Y et al. B cells suppress the inflammatory response in a mouse model of primary biliary cirrhosis. Gastroenterology. 2009; 136:1037-47.

102. Matsushita T, Horikawa M, Iwata Y, Tedder TF. Regulatory B cells (B10 cells) and regulatory T cells have independent roles in controlling experimental autoimmune encephalomyelitis initiation and latephase immunopathogenesis. J Immunol. 2010;185:2240-52.

103. Lee $\mathrm{KM}$ et al. Anti-CD45RB/anti-TIM-1-induced tolerance requires regulatory B cells. Am J Transplant. 2012;12:2072-8.

104. Miles K et al. A tolerogenic role for Toll-like receptor 9 is revealed by B-cell interaction with DNA complexes expressed on apoptotic cells. Proc Natl Acad Sci U S A. 2012;109:887-92. This study demonstrates that apoptotic cells induce Bregs through a process that invovles the BCR and TLR-9.

105. Kobayashi N et al. TIM-1 and TIM-4 glycoproteins bind phosphatidylserine and mediate uptake of apoptotic cells. Immunity. 2007;27:927-40.

106.• Yoshizaki A et al. Regulatory B cells control T-cell autoimmunity through IL-21-dependent cognate interactions. Nature. 2012;491: 
264-8. This study demonstrates that Bregs may be expanded ex vivo for therapuetic purposes.

107. Gagliani $\mathrm{N}$ et al. Rapamycin combined with anti-CD45RB mAb and IL-10 or with G-CSF induces tolerance in a stringent mouse model of islet transplantation. PLoS One. 2011;6:e28434.

108. Salvalaggio PR et al. Antigen exposure during enhanced CTLA-4 expression promotes allograft tolerance in vivo. J Immunol. 2006; 176:2292-8.

109. Deng $\mathrm{S}$ et al. Cutting edge: transplant tolerance induced by antiCD45RB requires B lymphocytes. J Immunol. 2007;178: 6028-32.

110. Lal G, et al. B Cell Depletion Prevents Costimulatory Blockade Induced Tolerance. (Abstract). American Journal of Transplantation 10 Supplement s4, 182 (2010).

111.• Shen P et al. IL-35-producing B cells are critical regulators of immunity during autoimmune and infectious diseases. Nature. 2014;507:366-70. This paper identifies plasmablasts as the major in-vivo source of IL-35, a key new "B cell" regulatory cytokine.

112. Bouaziz JD, Yanaba K, Tedder TF. Regulatory B cells as inhibitors of immune responses and inflammation. Immunol Rev. 2008;224: 201-14.

113. Thaunat O, Morelon E, Defrance T. Am"B"valent: anti-CD20 antibodies unravel the dual role of $\mathrm{B}$ cells in immunopathogenesis. Blood. 2010;116:515-21.

114. Clatworthy MR et al. B-cell-depleting induction therapy and acute cellular rejection. N Engl J Med. 2009;360:2683-5.
115. Bouaziz JD et al. IL-10 produced by activated human B cells regulates $\mathrm{CD} 4(+)$ T-cell activation in vitro. Eur J Immunol. 2010;40:2686-91.

116. Iwata $\mathrm{Y}$ et al. Characterization of a rare IL-10-competent B-cell subset in humans that parallels mouse regulatory B10 cells. Blood. 2011;117:530-41. This study serves as a counterbalance to previous studies and shows that memory B cells secrete significant amounts of IL-10, and that this may be increased in autoimmune patients.

117. Blair PA et al. CD19(+)CD24(hi)CD38(hi) B Cells Exhibit Regulatory Capacity in Healthy Individuals but Are Functionally Impaired in Systemic Lupus Erythematosus Patients. Immunity. 2010;32:129-40.

118. Newell KA et al. Identification of a B cell signature associated with renal transplant tolerance in humans. J Clin Invest. 2010;120: 1836-47.

119. Sagoo P et al. Development of a cross-platform biomarker signature to detect renal transplant tolerance in humans. J Clin Invest. 2010;120:1848-61.

$120 . \bullet$ Cherukuri A, et al. Immune regulation by human B cell subsets is influenced by the balance of IL-10 and TNF. J Am Soc Nephrol. In Press(2014). This study shows that human Breg activity correlates best with IL-10:TNFa ratio in transitional B cells, and that this ratio falls during rejection and may predict bad three-year outcomes.

121. Bar-Or A et al. Abnormal B-cell cytokine responses a trigger of Tcell-mediated disease in MS? Ann Neurol. 2010;67:452-61. 